vaccines, microbicides, barrier methods, circumcision and behavioural prevention interventions, and assessed possible factors associated with success and failure to achieve anticipated outcomes.

Results Of the hundreds of prevention trials conducted over the past decade, only six reported significant findings, of which three were male circumcision trials, with one each for prophylactic vaccines, Truvada for PREP, and TDF+FTC microbicide gel. Most biomedical prevention trials rely on patient reports of adherence to use, and generally adherence is significantly lower than advised. Condom use among treatment arms are also usually lower than in control arms, which when linked with insufficient product use, leads to increased risk of disease acquisition. Repeated behavioural prevention counselling, HIV testing, STD detection and treatment all lead to diminished reports of risk behaviour among both intervention and control participants, diminishing the statistical power of the planned trials. IRB requirements may lead to control conditions that provide prevention services dramatically greater than is involved in "usual care". RCTs which randomise individuals can also lead to significant cross-exposure among treatment arms.

Conclusions Alternative to the RCT Conclusions: Design alternatives to the individual or community randomised controlled trial may be required in the future. Increased attention to improving adherence to recommended use of prevention approaches which must repeatedly conducted (eg, use of a microbicide before each sex act), and alternatives to enriching control conditions above that of usual care should be considered, while maintaining fidelity to ethical conduct of research.

\section{P1-S6.04 EDUCATION AND LIFETIME RISK OF HIV INFECTION IN MANICALAND, ZIMBABWE}

doi:10.1136/sextrans-2011-050108.228

\begin{abstract}
${ }^{1} \mathrm{~L}$ Robertson, ${ }^{2} \mathrm{P}$ Mushati, ${ }^{1} \mathrm{~J}$ Eaton, ${ }^{1} \mathrm{C}$ Schumacher, ${ }^{2,3} \mathrm{~S}$ Gregson, ${ }^{2} \mathrm{C}$ Nyamukapa. ${ }^{1}$ Imperial College London, London, UK; ${ }^{2}$ Biomedical Research \& Training Institute Zimbabwe, Zimbabwe; ${ }^{3}$ Imperial College London, UK
\end{abstract}

Background There is evidence that attendance at school is protective against HIV among young people in South Africa. Further work is required to investigate the effect of education on the life-time risk of HIV in sub-Saharan Africa. In this paper, we have examined the association between education and HIV infection/sexual risk behaviour among men and women of different ages in Zimbabwe. Methods The effects of education level (none or primary only; secondary or higher) on prevalent HIV infection and sexual risk behaviours among young people of school age (15-21 years) and older adults (22-54 years) were investigated using data from four rounds of an open cohort study collected between 1999 and 2008 in Manicaland, Eastern Zimbabwe. Multivariate regression models were used to investigate the independent effects of education, marriage and wealth

Results School-aged men, interviewed in 1999/2000, with secondary or higher education had significantly reduced risk of HIV infection compared to those with none or primary education only (adjusted $\mathrm{OR}=0.35 ; \mathrm{p}=0.011$ ). For older men, the effect of education level was not significantly associated with HIV risk (adjusted $\mathrm{OR}=1.17$; $p=0.125)$. For those interviewed in $2006 / 2008$, education level was not found to be independently associated with HIV infection among school-aged or older men. At both time points, marital status and living in a town were independent risk factors for HIV among older men. Among women interviewed in 1999/2000, adjusted models indicate that a higher level of education had a significant protective effect for school-aged (adjusted $\mathrm{OR}=0.64 ; \mathrm{p}=0.023$ ) and older (adjusted $\mathrm{OR}=0.80 ; \mathrm{p}=0.017$ ) women. For those interviewed in $2006 / 2008$, a higher level of education level was protective among school-aged (adjusted $\mathrm{OR}=0.56 ; \mathrm{p}=0.040$ ) but not older (adjusted $\mathrm{OR}=0.99 ; \mathrm{p}=0.940$ ) women. Marital status was an independent risk factors for HIV infection fort women of all ages.
Conclusions There is evidence that secondary or higher education is protective against HIV infection among school-aged people in Zimbabwe, particularly women. Among older men, education does not appear to be protective against HIV. In 1999/2000, secondary or higher education was protective against HIV among older women. However, in 2006/2008, this protective effect was reduced. Further work to calculate the lifetime cumulative risk of HIV infection by education level, in this population, will be conducted.

\section{P1-S6.05 INFLUENCE OF SOCIAL SUPPORT NETWORKS ON THE HIV TRANSMISSION RISK BEHAVIOURS OF PEOPLE LIVING WITH HIV IN MANITOBA, CANADA}

doi:10.1136/sextrans-2011-050108.229

\section{A Cameron. University of Manitoba, Winnipeg, Canada}

For people living with HIV/AIDS (PLWHA) positive social support networks (SSNs) can help improve quality of life, overall well-being, coping, and decrease mood disturbance, morbidity, mortality, sexual and substance associated HIV transmission risk factors. However, HIV diagnosis can cause a negative change in SSNs leading to social isolation (actual/perceived) and increase risk of HIV transmission behaviours. Having an effective strategy to encourage the development/maintenance of SSNs may have a positive effect upon the health outcomes and HIV transmission risk behaviours of PLWHA. Objective To describe the SSNs of Manitobans living with HIV/ AIDS (MLHA) and determine the influence of SSNs on transmission risk behaviour. The relationship between independent variable (size and type of SSNs- positive/negative) and dependant variables (sexual risk behaviour, and alcohol, injection and non-injection drug use) was examined. Control variables included: age, gender, ethnicity, time since diagnosis, and sexual orientation. This data was collected in the Positive Prevention Study (PPS), a cross-sectional survey which included 135 MLHA aged 18 plus. The PPS assessed a broad list of transmission related determinants and only enrolled people if they were aware of their HIV diagnosis for at least 6 months, allowing for analysis of sustained positive behavioural changes. For this analysis SAS statistical software was used. Analysis of variance was done between the size and type (positive/ negative) of SSNs and the chosen transmission risk behaviours; sexual behaviour, alcohol use, injection and non-injection drug use. Analysis of covariance was conducted with independent, dependent and control variables. Multiple regression analysis was run with independent and dependent variables to determine any relation. Level of social support achievable depends on one's attachment to those in their SSN and the role they play. It is not just the quantity of people but also the quality of relationships (eg, frequency, perceived support) that defines the success of SSNs. Not all SSNs are positive; some types may increase transmission risk behaviour. Only positive SSNs (regardless of size) are associated with avoidance of transmission risk behaviours. The results of this study help to assess the degree to which SSNs affect the sustainability of long-term secondary prevention measures, and thus inform groups offering services to MLHA with local scientific evidence.

\section{P1-S6.06 WHAT HAS BEEN THE EFFECT OF THE AVAHAN HIV INTERVENTION ON CONDOM USE AMIONG FEMALE SEX WORKERS? INSIGHTS FROM A FULLY BAYESIAN INFERENCE METHODOLOGY}

doi:10.1136/sextrans-2011-050108.230

${ }^{1} \mathrm{~J}$ Dureau, ${ }^{2} \mathrm{M}$ C Boily, ${ }^{3} \mathrm{P}$ Vickerman, ${ }^{2} \mathrm{M}$ Pickles, ${ }^{4} \mathrm{~S}$ Isac, ${ }^{4} \mathrm{~B}$ Ramesh, ${ }^{4} \mathrm{~S}$ Verma,

${ }^{5} \mathrm{~S}$ Moses, ${ }^{6} \mathrm{M}$ Alary, ${ }^{1} \mathrm{~K}$ Kalogeropoulos. ${ }^{1}$ London School of Economics, London, UK; ${ }^{2}$ Imperial College, UK; ${ }^{3}$ London School of Hygiene \& Tropical Medicine, UK; ${ }^{4}$ Karnataka Health Promotion Trust, Bangalore, India; ${ }^{5}$ University of Manitoba, Winnipeg, Canada; ${ }^{6}$ Universite Laval, Canada 